\title{
Efficacy of Periportal Infiltration and Intraperitoneal Instillation of Ropivacaine After Laparoscopic Surgery in Children
}

\author{
Maria Rita Di Pace, MD, ${ }^{1}$ Marcello Cimador, MD, ${ }^{1}$ Pieralba Catalano, MD, ${ }^{1}$ Anna Caruso, MD, ${ }^{1}$ \\ Maria Sergio, MD, ${ }^{1}$ Alessandra Casuccio, MD, ${ }^{2}$ and Enrico De Grazia, MD, ${ }^{1}$
}

\begin{abstract}
Postoperative pain is less intense after laparoscopic than after open surgery. However, minimally invasive surgery is not a a pain-free procedure. Many trials have been done in adults using intraperitoneal and/or incisional local anesthetic, but similar studies have not yet been reported in the literature in children.

Aim: The aim of this study was to evaluate the analgesic effect of periportal infiltration and intraperitoneal instillation of ropivacaine in children undergoing laparoscopic surgery.

Materials and Methods: Thirty patients who underwent laparoscopic surgery were randomly allocated to one of three groups. Group A $(n=10)$ received local infiltration of port sites with $10 \mathrm{~mL}$ of ropivacaine. Group B $(n=10)$ received both an infiltration of port sites with $10 \mathrm{~mL}$ of ropivacaine and an intraperitoneal instillation of $10 \mathrm{~mL}$ of ropivacaine. Group $\mathrm{C}$ did not receive any analgesic treatment. The local anesthetic was always administered at the end of surgery. The degree of postoperative abdominal parietal pain, abdominal visceral pain, and shoulder pain was assessed by using a Wong-Baker pain scale and a Visual Analog Scale (VAS) at 3, 6 12, and 24 hours postoperatively. The following parameters were also evaluated: rescue analgesic treatment, length of hospital stay, and time of return to normal activities.

Results: Three hours after operation, patients had low pain scores. Six and 12 hours postoperatively, the abdominal parietal pain was significantly higher $(P<0.0005)$ in group $C$ than in the other two groups, both treated with an infiltration at the trocar sites; mean intensity of abdominal visceral pain was significantly lower $(P<$ $0.0005)$ in group B than in groups A and C; the overall incidence of shoulder pain was significantly lower $(P<$ 0.0005 ) in group B patients than in patients of groups A and C. At 20 hours postoperatively, pain scores were significantly reduced of intensity in all groups. Rescue analgesic treatment was significantly higher in group C, if compared to groups A and B 12 hours after the operation. No statistically significant difference was found in length of hospital stay, but children who received analgesic treatment had a more rapid return to normal activities than untreated patients $(P<0.0005)$.

Conclusions: Our study demonstrates that the combination of local infiltration and intraperitoneal instillation of ropivacaine is more effective for pain relief in children after laparoscopic surgery than the administration of ropivacaine only at the trocar sites.
\end{abstract}

\section{Introduction}

$\mathbf{P}$ OSTOPERATIVE PAIN is less intense after minimally invasive surgery than after open surgery. However, laparoscopy is not a pain-free procedure and the management of postlaparoscopy pain remains a major concern. Actually, it has been reported that from 35 to $63 \%$ of patients undergoing laparoscopic surgery suffer pain, mainly during the first postoperative hours. ${ }^{1}$ The origin of pain after laparoscopic procedures is multifactorial, with pain arising from the incisional trauma at port sites, the distention and chemical irritation of the peritoneum, the diaphragmatic stretching with phrenic nerve neuropraxia, and direct tissue injury. ${ }^{2-4}$ Many trials have been done in adults using intraperitoneal and/or incisional local anesthetic, but, to our knowledge, similar studies have not yet been reported in the literature in pedi-

Pediatric Surgical Unit, Departments of ${ }^{1}$ Mother and Child Care and ${ }^{2}$ Clinical Neuroscience, Università di Palermo, Palermo, Italy. 
atric age. 5,6 The aim of this study was to determine whether periportal infiltration and intraperitoneal instillation of ropivacaine would reduce postoperative pain in children who undergo laparoscopic procedures.

\section{Materials and Methods}

This study was approved by our local ethics committee, and a preoperative informed consent for participation in the trial was obtained by parents. Thirty children, who underwent laparoscopic surgery from May 2006 to May 2007, were recruited. Exclusion criteria were: age $<6$ years, American Society of Anesthesiology (ASA) grade 3 or more, contraindications to any of the drugs used in the study, previous abdominal surgery, and operating time $>120$ minutes. The laparoscopic procedures included: cholecystectomy, appendectomy, transperitoneal Palomo, ovarian surgery, and other procedures for chronic pelvic pain, Morris syndrome, Turner syndrome, and other defects of sexual differentiation.

All children received premedication with midazolam 0.15 $\mathrm{mg} / \mathrm{kg}$ intramuscularly (i.m.). General anesthesia was inducted with thiopental $5 \mathrm{mg} / \mathrm{kg}$, atracurium $0.5 \mathrm{mg} / \mathrm{kg}$, and fentanyl $2 \gamma / \mathrm{kg}$ intravenously (i.v.). Anesthesia was maintained with a sevoflurane and oxygen mixture. The pneumoperitoneum was established via open laparoscopy, following the Hasson technique. In addition, two or three trocars ( 3 or $5 \mathrm{~mm}$ ) were used.

Patients were randomly assigned to one of three groups. Group A $(n=10)$ received a local infiltration of $10 \mathrm{~mL}$ of ropivacaine at the port sites. Group B received an infiltration of trocar sites with $10 \mathrm{~mL}$ of ropivacaine and an intraperitoneal instillation of $10 \mathrm{~mL}$ of ropivacaine. Group C (control group) received no analgesic treatment. Ropivacaine (2 $\mathrm{mg} / \mathrm{mL}$ ) was used for children under 10 years of age and $7.5 \mathrm{mg} / \mathrm{mL}$ for patients aged 10 years or more. The local anesthetic was always administered at the end of surgery. For the intraperitoneal infusion, solution was instillated under each subdiaphragmatic space through a suction-irrigation device under visual control. Intra-abdominal pressure was maintained at 10 or $12 \mathrm{~mm} \mathrm{Hg}$ on the basis of age (respectively, $<10$ and $\geq 10$ years). At the end of the procedure, $\mathrm{CO}_{2}$ was accurately removed from the peritoneal cavity by manual compression of the abdomen.

Before surgery, patients and parents were instructed in the use of two pain scales: the Wong-Baker scale and the $10-\mathrm{cm}$ Visual Analog Scale (VAS), both with scores raging from 0 (no pain) to 10 (worst possible pain). The degree of postoperative abdominal parietal pain (APP), defined as abdomi- nal wall incisional pain, abdominal visceral pain (AVP), defined as deep in the abdomen with poor localization, and shoulder tip pain (STP) was assessed by using the pain scales at $3,6,12$, and 24 hours postoperatively. For postoperative analgesia, children with a pain score $\geq 6$ were given analgesic drugs, namely paracetamol + codeine immediate release $(200+5 \mathrm{mg})$, if aged less than 10 years, and ketorolac i.v. $(0.4 \mathrm{mg} / \mathrm{kg})$, if aged 10 years or more. The following parameters were also evaluated: rescue analgesic treatment, length of hospital stay, and time of return to normal activities.

\section{Statistical analysis}

Data are expressed as the mean \pm standard deviation. Frequency analysis was performed with the chi-squared test. The univariate analysis of variance test and the Kruskal-Wallis test were used for parametric and nonparametric analysis, respectively, to evaluate differences between the groups. All $P$-values were two-sided and $P$-values less than 0.05 were considered to indicate statistical significance. Data were analyzed by EpiInfo (version 6.0; Centers for Disease Control and Prevention, Atlanta, GA) and SPSS software (version 14.0; SPSS Inc., Chicago, IL).

\section{Results}

Thirty patients were randomized to three groups equal in size $(n=10)$. There were no significant differences between the groups with respect to age, sex, body mass index (Table 1 ), and operating time. No conversion to open surgery was necessary for any patient. No intraoperative complications were recorded. In all children there was correspondence between the scores given by the Wong-Baker scale and the VAS.

Postoperative pain scores are shown in Table 2. Three hours after the operation, patients did not differ significantly in shoulder pain intensity, but they had a significant difference in APP and AVP ( $P=0.002$ and 0.022 , respectively). Group B children, who had received both local infiltration and intraperitoneal instillation of ropivacaine, had lower pain scores. At the 6- and 12-hour postoperative evaluations, the mean intensity of AVP was significantly lower $(P<0.0005)$ in group $B$ than in group $A$ and $C$ patients; APP was significantly higher $(P<0.0005)$ in group $C$ patients than if compared with the other two groups, both treated with infiltration at the trocar sites; the overall incidence of shoulder pain in group B patients was significantly lower $(P<0.0005)$ than that recorded in group $\mathrm{A}$ and $\mathrm{C}$ patients. These differences

Table 1. Patient Characteristics

\begin{tabular}{lcccc}
\hline $\begin{array}{l}\text { Characteristic } \\
\text { value }\end{array}$ & $\begin{array}{c}\text { Group A } \\
(n=10)\end{array}$ & $\begin{array}{c}\text { Group B } \\
(n=10)\end{array}$ & $\begin{array}{c}\text { Group C } \\
(n=10)\end{array}$ & $P$ - \\
\hline $\begin{array}{l}\text { Age (years) } \\
\text { Gender }(\mathrm{M} / \mathrm{F})\end{array}$ & $\begin{array}{c}11.3 \pm 3.0 \\
4 / 6\end{array}$ & $\begin{array}{c}10.8 \pm 3.2 \\
5 / 5\end{array}$ & $\begin{array}{c}12.3 \pm 3.2 \\
6 / 4\end{array}$ & 0.562 \\
BMI (percentile) & $41.2 \pm 19.8$ & $43.1 \pm 22.1$ & $40.9 \pm 27.2$ & 0.670 \\
\hline
\end{tabular}

Group A = local infiltration at port sites; group B = local infiltration + intraperitoneal instillation; group $\mathrm{C}=$ no analgesic treatment. 
Table 2. VAS/Wong-Baker Scores in the Three Groups

\begin{tabular}{llllc}
\hline Kind of pain & $\begin{array}{l}\text { Group } A \\
(n=10)\end{array}$ & $\begin{array}{l}\text { Group B } \\
(n=10)\end{array}$ & $\begin{array}{l}\text { Group C } \\
(n=10)\end{array}$ & P-value \\
\hline APP (hours) & & & & \\
3 & $0.9 \pm 0.74$ & $0.5 \pm 0.71$ & $1.9 \pm 0.74$ & 0.002 \\
6 & $1.8 \pm 0.92$ & $1.1 \pm 0.88$ & $5.2 \pm 1.55$ & $<0.0005$ \\
12 & $2.8 \pm 0.9$ & $1.8 \pm 0.92$ & $7.2 \pm 1.23$ & $<0.0005$ \\
24 & $2.0 \pm 1.25$ & $1.7 \pm 0.82$ & $3.0 \pm 1.05$ & 0.035 \\
AVP (hours) & & & & \\
3 & $2.2 \pm 1.13$ & $1.4 \pm 0.52$ & $2.5 \pm 0.85$ & 0.022 \\
6 & $5.1 \pm 1.73$ & $1.8 \pm 1.03$ & $6.0 \pm 0.94$ & $<0.0005$ \\
12 & $4.7 \pm 0.95$ & $1.3 \pm 0.67$ & $5.3 \pm 0.67$ & $<0.0005$ \\
24 & $0.3 \pm 0.48$ & $0.3 \pm 0.48$ & $0.4 \pm 0.52$ & 0.865 \\
STP (hours) & & & & 0.686 \\
3 & $0.8 \pm 0.79$ & $0.7 \pm 0.67$ & $1.0 \pm 0.82$ & $<0.0005$ \\
6 & $3.0 \pm 1.94$ & $0.9 \pm 0.87$ & $3.7 \pm 0.82$ & $<0.0005$ \\
12 & $4.1 \pm 1.29$ & $1.4 \pm 0.84$ & $4.0 \pm 0.82$ & $<0.039$ \\
24 & $0.1 \pm 0.32$ & $0.1 \pm 0.32$ & $0.3 \pm 0.48$ & 0.395 \\
\hline
\end{tabular}

Data are expressed as the mean \pm standard deviation.

APR, abdominal parietal pain; AVP abdominal visceral pain; STP, shoulder tip pain.

among the groups became nonsignificant at 24 hours postoperatively, except for APP $(P=0.035)$. Actually, AVP and STP were almost absent, whereas APP was lower, but persisted until 36 hours, causing discomfort in children (Fig. 1).

The number of patients who required analgesic treatment in the postoperative time was significantly higher $(P=0.001)$ in group C, if compared to groups A and B 12 hours after the operation ( 8 vs. 4 and 0 patients, respectively). No child of group B required analgesic drugs during postoperative time (Table 3). No statistically significant difference was found in length of hospital stay $(P=0.827)$, but children who received local analgesic treatment had a more rapid return
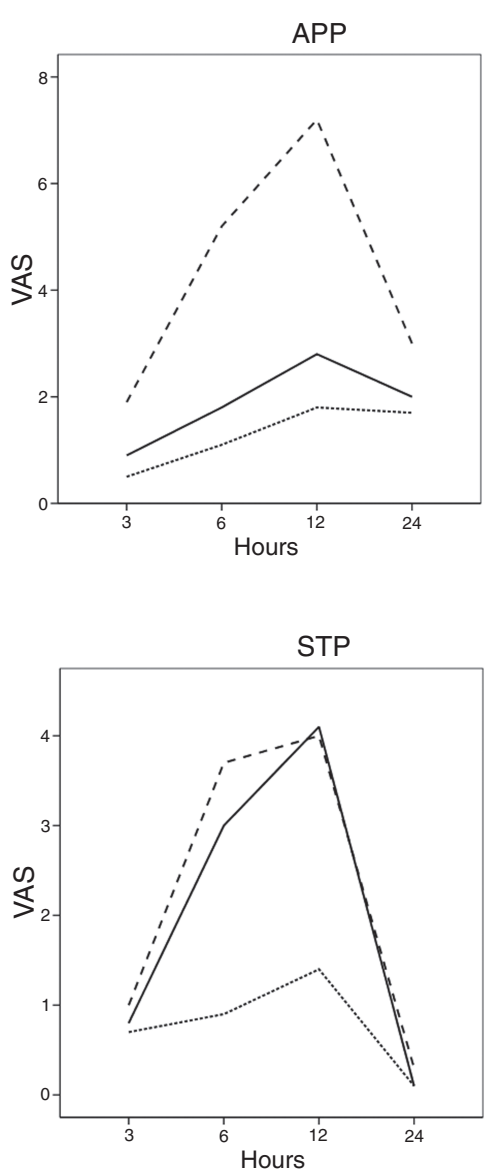
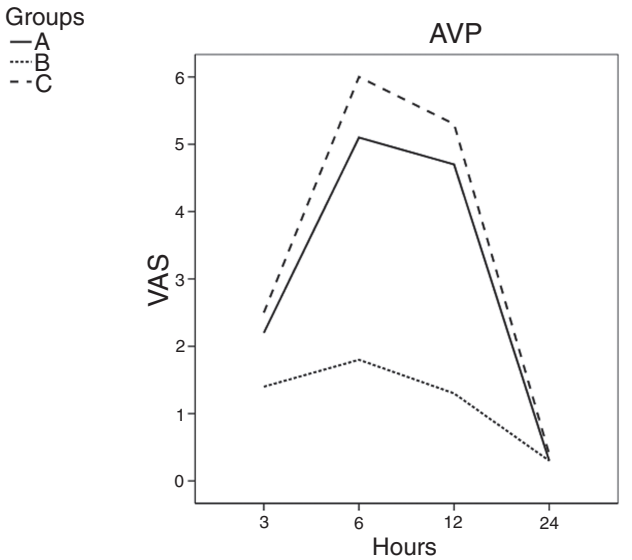

Groups<smiles>C1=C[GeH]C1</smiles>

Groups

$-A$
$-\cdots \cdot B$
$--C$
FIG. 1. Intensity of abdominal parietal pain (APP), abdominal visceral pain (AVP), and shoulder tip pain (STP) recorded on the VAS/Wong-Baker pain scales at 3, 6, 12, and 24-hour postoperative evaluations for groups A (local infiltration at port sites), B (local infiltration + intraperitoneal instillation), and $C$ (no analgesic treatment). 
TAble 3. Intraoperative ANd Postoperative VARIAbles

\begin{tabular}{|c|c|c|c|c|}
\hline Variable & Group A & Group B & Group C & p-value \\
\hline Operating time (minutes) ${ }^{\mathrm{a}}$ & $60.5 \pm 25.1$ & $72.5 \pm 24.9$ & $62.0 \pm 24.1$ & 0.504 \\
\hline $\begin{array}{l}\text { Rescue analgesic treatment at } 6 \text { hours } \\
\text { (number of patients) }\end{array}$ & $6 / 10$ & $0 / 10$ & $7 / 10$ & 0.003 \\
\hline $\begin{array}{l}\text { Rescue analgesic treatment at } 12 \text { hours } \\
\text { (number of patients) }\end{array}$ & $4 / 10$ & $0 / 10$ & $8 / 10$ & 0.001 \\
\hline Time of return to normal activities (hours) ${ }^{\mathrm{a}}$ & $9.6 \pm 3.09$ & $7.8 \pm 2.89$ & $22.8 \pm 10.51$ & $<0.0005$ \\
\hline Hospitalization (hours) ${ }^{\mathrm{a}}$ & $38.4 \pm 16.78$ & $40.8 \pm 19.76$ & $43.2 \pm 15.18$ & 0.827 \\
\hline
\end{tabular}

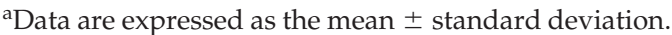

to normal activities than untreated patients (group $\mathrm{A}=9.6$ and group $\mathrm{B}=7.8$ hours vs. group $\mathrm{C}=22.8$ hours; $P<$ 0.0005) (Table 3).

\section{Discussion}

Pain during the first postoperative hours remains the most prevalent complaint after laparoscopic surgery. Many factors can be responsible for the pain; namely, abdominal distention, chemical irritation of the peritoneum, phrenic nerve neuropraxia secondary to diaphragmatic stretching, operative tissue damage, or incisional trauma at the trocar sites. ${ }^{8}$ The pain associated with laparoscopic procedures has two main components: a visceral component, caused either by the surgical manipulation or by diaphragmatic stretching and irritation from $\mathrm{CO}_{2}$, and a somatic one, related to incisional trauma at port sites. ${ }^{6}$

Different methods have been used in the adult population in an attempt at reducing the intensity of pain after minimally invasive surgery, including a low-pressure pneumoperitoneum, periportal anesthetic infiltration, saline washout, and intraperitoneal instillation of local anestethics. ${ }^{2,9}$ Similar studies have not yet been reported in the literature in children. Local anesthetics induce antinociception by inhibiting the release and action of proteolitic and inflammatory agents, such as histamine, serotonin, bradykinin, and prostaglandins. These agents are released into tissues after a surgical injury and stimulate nociceptors, thus activating and maintaining postoperative pain. The efficacy of local anesthetic agents has been demonstrated in laparoscopic cholecystectomy ${ }^{10}$ and in gynecologic surgery in adult patients. ${ }^{11}$ Ropivacaine, a long-acting local anesthetic, has proved to be less likely than other drugs to elicit adverse effects from the central nervous system and the circulatory system ${ }^{12}$ and was, therefore, chosen in our study concerning a pediatric population.

Our data clearly show that patients who received both a local anesthetic infiltration of port sites and intraperitoneal instillation of ropivacaine suffer a significantly less intense parietal, visceral, and shoulder tip pain, if compared to patients who received no local analgesic treatment. However, this former group had been immediately treated for VAS score $\geq 6$. Further, a significant difference was found between groups A and B as for visceral and shoulder tip pain, but not for parietal pain, due to the local anesthetic infiltration of port sites performed in both groups. However, parietal pain reached a higher intensity in group $A$ than in group $B$, probably because the lower perception of visceral and/or shoul- der tip pain in the latter group, due to intraperitoneal instillation, also influenced the parietal pain.

As for analgesic consumption, our study clearly shows that group $C$ patients requested additional analgesic administration, always after the 3rd postoperative hour and never 24 hours after the operation, reaching a peak at the 12hour evaluation. The analgesia request rate was lower in group A than in group C patients, but the peak of patients requesting additional analgesic administration was recorded at the 6-hour evaluation. Our study shows that in both groups, the analgesia consumption curve followed the intensity of pain curve progress. Actually, in group C, the pain curve increased gradually, with a peak 12 hours after the operation, due to parietal pain and, afterward decreased promptly. Instead, in group A patients, parietal pain was tolerable, due to port-site local anesthetic infiltration, whereas visceral pain was predominant, with the higher intensity recorded at the 6-hour evaluation and a gradual decrease during the following hours. Group B patients requested no analgesic administration, demonstrating that the combined treatment was effective. Namely, the intraperitoneal instillation of ropivacaine proved to be successful in reducing both visceral and shoulder tip pain, thus allowing patients to endure parietal pain.

Our results show that the combination of local infiltration and intraperitoneal instillation of ropivacaine immediately after laparoscopic procedures is effective in reducing postoperative abdominal and shoulder pain in children. Some trials have suggested that preemptive analgesia provides a greater reduction of postoperative pain than the peri- or postoperative administration of local anesthetic agents in adults. Actually, many researchers stress that the timing of administration is essential for the reduction of postoperative pain. However, the effects of pre- and postincisional infiltration of the surgical area on cortisol and prolactin release and postoperative pain in chidren undergoing inguinal hernia repair have been studied and no statistically significant differences were found. ${ }^{12}$

Hence, we emphasize the need for a larger multicentric study on local anaesthetics administrated either preoperatively or postoperatively, in order to evaluate possible differences in terms of reduction of postoperative pain and to implement a technique as a standard step during laparoscopic procedures in children.

\section{Disclosure Statement}

No competing financial interests exist. 


\section{References}

1. Slim K. Pain after laparoscopic cholecystectomy. Br J Surg 2000;87:1249.

2. Barczynski M, Konturek A, Herman RM. Superiority of preemptive analgesia with intraperitoneal instillation of bupivacaine before, rather than after, the creation of pneumoperitoneum for laparoscopic cholecystectomy: A randomized, double-blind, placebo-controlled study. Surg Endosc 2006;20:1088-1093.

3. Mouton WG, Bessell JR, Otten KT, Maddern GJ Pain after laparoscopy. Surg Endosc 1999;13:445-448.

4. Wills VL, Hunt DR. Pain after laparoscopic cholecystectomy. Br J Surg 2000;87:273-284.

5. Papagiannopoulou P, Argiriadou H, Georgiou M, Papaziogas B, Sfyra E, Kanakoudis F. Preincisional local infiltration of levobupivacaine vs. ropivacaine for pain control after laparoscopic cholecystectomy. Surg Endosc 2003;17:1961-1964.

6. Chou YJ, Ou YC, Lan KC, Jawan B, Chang SY, Kung FT. Preemptive analgesia instillation during gynaecologic laparoscopy: A randomized trial. J Min Invas Gynecol 2005;12: 330-335.

7. Ivani G. Ropivacaine: Is it the time for children? Paediatr Anaesth 2002;12:383-387.

8. Louizos AA, Hadzilia SJ, Leandros E, Kouroukli IK, Georgiou LG, Bramis JP. Postoperative pain relief after laparoscopic cholecystectomy. Surg Endosc 2005;19:1503-1506.

9. Barczynski M, Herman RM. Low-pressure pneumoperitoneum combined with intraperitoneal saline washout for reduction of pain after laparoscopic cholecystectomy: A prospective, randomized study. Surg Endosc 2004;18:13681373.

10. Paulson J, Mellinger J, Baguley W. The use of intraperitoneal bupivacaine to decrease the length stay in elective laparoscopic cholecystectomy patients. Am Surg 2003;69: 275-278.

11. Shaw IC, Stevens J, Krishnamurthy S. The influence of intraperitoneal bupivacaine on pain following major laparoscopic gynaecological procedures. Anaesthesia 2001;56: 1041-1044.

12. Sakellaris G, Petrakis I, Makatounaki K, Arbiros I, Karkavitsas N, Charissis G. Effects of ropivacaine infiltration on cortisol and prolactin responses to postoperative pain after inguinal herniorraphy in children. I Ped Surg 2004;39:14001403.

Address correspondence to: Marcello Cimador, MD Dipartimento Universitario Materno-Infantile Università di Palermo Via Cardinale Rampolla, 1 90142, Palermo Italy

E-mail: mcimador@unipa.it 
